\title{
Investigation of the $\mathrm{CaO}-\mathrm{Al}_{2} \mathrm{O}_{3}-\mathrm{SiO}_{2}-\mathrm{CaSO}_{4}-$ $\mathrm{CaCO}_{3}-\mathrm{H}_{2} \mathrm{O}$ system at $25^{\circ} \mathrm{C}$ by thermodynamic calculation
}

\author{
D. Damidot,* S. J. Barnett, † F. P. Glasser $\ddagger$ and D. E. Macphee
}

Ecole des Mines de Douai; University of Liverpool; University of Aberdeen

The phase diagram of the $\mathrm{CaO}-\mathrm{Al}_{2} \mathrm{O}_{3}-\mathrm{SiO}_{2}-\mathrm{CaSO}_{4}-\mathrm{CaCO}_{3}-\mathrm{H}_{2} \mathrm{O}$ system relevant to cement has been calculated at $25^{\circ} \mathrm{C}$ and 1 bar pressure. It is composed of 331 stable phase assemblages in equilibrium with the aqueous phase and contains 30 invariant points with 15 solids. Thaumasite appears to be a stable phase with a wide stability domain. Compared to ettringite, thaumasite is stable to lower $\mathrm{pH}$ and generally demands higher sulphate concentrations to be stable.

\section{Introduction}

Thaumasite $\left(\mathrm{Ca}_{6} \mathrm{Si}_{2}\left(\mathrm{SO}_{4}\right)_{2}(\mathrm{OH})_{12}\left(\mathrm{CO}_{3}\right)_{2} \cdot 24 \mathrm{H}_{2} \mathrm{O}\right)$ and ettringite $\left(\mathrm{Ca}_{6} \mathrm{Al}_{2}\left(\mathrm{SO}_{4}\right)_{3}(\mathrm{OH})_{12} \cdot 26 \mathrm{H}_{2} \mathrm{O}\right)$ formation in mortars and concrete are related to the interaction of aqueous sulphates and carbonates with the solid components of cement matrices. However, the exact conditions under which the thaumasite form of sulphate attack (TSA) occurs are still not fully known, mainly due to the lack of basic knowledge on thaumasite such as its temperature range of stability, its $\mathrm{pH}$ stability and the nature of other phases compatible with thaumasite. Thaumasite is generally considered to need low temperatures to form at appreciable reaction rates; ${ }^{1}$ however, field occurrences of thaumasite have been reported in areas known for their mild climates. ${ }^{2}$

The main objective of this work consists of a thermodynamic investigation of the $\mathrm{CaO}-\mathrm{Al}_{2} \mathrm{O}_{3}-\mathrm{SiO}_{2}-$ $\mathrm{CaSO}_{4}-\mathrm{CaCO}_{3}-\mathrm{H}_{2} \mathrm{O}$ system at $25^{\circ} \mathrm{C}$. This temperature has been selected because thermodynamic data are either available for $25^{\circ} \mathrm{C}$ or, as for thaumasite, can be extrapolated to $25^{\circ} \mathrm{C}$ using literature data. Also, several important constituent subsystems have already been investigated at $25^{\circ} \mathrm{C}: \mathrm{CaO}-\mathrm{Al}_{2} \mathrm{O}_{3}-\mathrm{CaSO}_{4}-\mathrm{H}_{2} \mathrm{O},{ }^{3} \mathrm{CaO}-$

\footnotetext{
* Ecole des Mines de Douai, Civil Engineering Department, 59000 Douai, France.

$\dagger$ Department of Civil Engineering, University of Liverpool, Liverpool L69 3GQ, UK.

$\ddagger$ Department of Chemistry, University of Aberdeen, Aberdeen AB9 2UE, Scotland, UK.
}

$\mathrm{Al}_{2} \mathrm{O}_{3}-\mathrm{CaSO}_{4}-\mathrm{CaCO}_{3}-\mathrm{H}_{2} \mathrm{O},{ }^{4}, \mathrm{CaO}-\mathrm{SiO}_{2}-\mathrm{CaSO}_{4}-\mathrm{H}_{2} \mathrm{O},{ }^{5}$ $\mathrm{CaO}-\mathrm{SiO}_{2}-\mathrm{CaCO}_{3}-\mathrm{H}_{2} \mathrm{O}$, ${ }^{5}$ and $\mathrm{CaO}-\mathrm{Al}_{2} \mathrm{O}_{3}-\mathrm{SiO}_{2}-$ $\mathrm{H}_{2} \mathrm{O}$. ${ }^{6}$ More complex systems have been partially studied such as the eight-component system $\mathrm{CaO}-\mathrm{Al}_{2} \mathrm{O}_{3}-\mathrm{SiO}_{2}-$ $\mathrm{Fe}_{2} \mathrm{O}_{3}-\mathrm{MgO}-\mathrm{CaSO}_{4}-\mathrm{CaCO}_{3}-\mathrm{H}_{2} \mathrm{O}{ }^{7}$ However, in this latter case the model did not include such important stable phases as hydrogarnet and hydrotalcite and, as a consequence, the results are incomplete.

\section{Method of calculation}

\section{Solubility determination}

It is necessary to know the solubility product of thaumasite in order to determine the stability conditions of thaumasite by thermodynamic calculations. Thaumasite was first synthesised and then redispersed in water at different temperatures ranging from 5 to $30^{\circ} \mathrm{C}$, as reported by Edge and Taylor. ${ }^{8}$ Analyses of constituent ion concentrations after several redispersions of thaumasite were obtained by standard analytical chemical techniques. Thaumasite and ettringite can each undergo ionic substitutions and a partial solid solution exists in the thaumasite-ettringite system. Although ettringite and thaumasite are partially miscible with up to $50 \mathrm{~mol} \%$ of the thaumasite component soluble in ettringite, much less ettringite is soluble in thaumasite. The authors have therefore considered only the 'pure' thaumasite end member in this study. Data for the solid solution, showing negligible solid solution of ettringite in thaumasite but substantial solid solution in ettringite, will be reported in the near future. 


\section{Thermodynamic database}

In order to be consistent with the previous calculations obtained on the subsystems, the same methodology and the same computer program, PHRQPITZ, ${ }^{10}$ which calculates the activity coefficients using Pitzer's relations, ${ }^{11}$ has been used. The method is based on the minimisation of free energy and the calculation outputs include, among other data, the invariant points of the system, tabulation of solids present and the composition of the aqueous coexisting phase. The accuracy of the results mainly depends on the reliability of the equilibrium constants and inclusion of all relevant solids; missing solids not included in calculations obviously have an important effect on the results.

The $\mathrm{CaO}-\mathrm{SiO}_{2}-\mathrm{H}_{2} \mathrm{O}$ system is certainly one of the most difficult to model due to the variable composition and metastability of $\mathrm{C}-\mathrm{S}-\mathrm{H}$ relative to crystalline calcium silicate hydrates. Although recent progress has been made in a physico-chemical treatment of $\mathrm{C}-\mathrm{S}-$ $\mathrm{H}^{12}$, the authors have kept to the approach used to calculate the $\mathrm{CaO}-\mathrm{Al}_{2} \mathrm{O}_{3}-\mathrm{SiO}_{2}-\mathrm{H}_{2} \mathrm{O}$ system which models $\mathrm{C}-\mathrm{S}-\mathrm{H}$ in terms of two fixed compositions, designated $\mathrm{C}-\mathrm{S}-\mathrm{H}(\mathrm{SI})$ and $\mathrm{C}-\mathrm{S}-\mathrm{H}(\mathrm{SII}) .{ }^{13} \mathrm{C}-\mathrm{S}-\mathrm{H}(\mathrm{SI})$ has a low $\mathrm{C} / \mathrm{S}$ ratio (fixed $\mathrm{C} / \mathrm{S}$ corresponding to an average value of $1 \cdot 1$ ) and is stable in solution having lime concentrations typically lower than $20 \mathrm{mmol} / \mathrm{l}$ at $25^{\circ} \mathrm{C}$. On the other hand, $\mathrm{C}-\mathrm{S}-\mathrm{H}(\mathrm{SII})$ has a higher $\mathrm{C} / \mathrm{S}$ ratio, fixed at 1.8 and is stable in solutions with lime concentrations greater than $20 \mathrm{mmol} / 1$ at $25^{\circ} \mathrm{C}$. Similarly, the authors have modelled the hydrogrossularitegrossularite garnet solid solutions by designating two hydrogarnet compositions: ${ }^{14}$ a low $\mathrm{SiO}_{2}$-containing hydrogarnet ( $\mathrm{HG} \mathrm{Si} \mathrm{low;} \mathrm{C}_{3} \mathrm{AS}_{0 \cdot 3} \mathrm{H}_{5 \cdot 4}$ ); and a higher $\mathrm{SiO}_{2}$-containing hydrogarnet (HG $\mathrm{Si}$ high; $\left.\mathrm{C}_{3} \mathrm{AS}_{0 \cdot 8} \mathrm{H}_{4 \cdot 4}\right)$.

Knowledge of the thermodynamic properties of the constituent phases of cement systems is still far from perfect and the present set of calculations cannot be more accurate than the numerical values of the database used in the calculation. However, the authors have taken account of the variation in composition of the $\mathrm{C}-$ $\mathrm{S}-\mathrm{H}$ phase as well as that of the hydrogarnet phase insofar as data are presently available with which to assess the relevant thermodynamic values. The consequences of the simplifications employed are discussed subsequently.

The values of the solubility products are not given here because they mainly depend on the method used to calculate them; for example, the species used to write the equation of dissolution, methods used to calculate activity coefficients. However, the composition of the solution in equilibrium with each solid used in this work is given in Table 1 and with these data the reader can, if desired, calculate a solubility product appropriate to a particular system by defining solubility equations and activity corrections.

\section{Results}

The calculated $\mathrm{CaO}-\mathrm{Al}_{2} \mathrm{O}_{3}-\mathrm{SiO}_{2}-\mathrm{CaSO}_{4}-\mathrm{CaCO}_{3}-$ $\mathrm{H}_{2} \mathrm{O}$ system at $25^{\circ} \mathrm{C}$ contains 30 invariant points (Table 2). It is difficult to represent graphically this phase diagram due to its high dimensionality. In order to describe it as accurately as possible, the stable phase assemblages deriving from the invariant points have been listed. The system has 331 stable phase assemblages in equilibrium with the aqueous phase, comprising 99 assemblages of four solids (Table 3), 121 assemblages of three solids (Table 4), 66 assemblages of two solids (Table 5) and finally 15 stable solids. It is also of interest to know which phases are not stable together. Key assemblages of two phases that are not compatible are listed in Table 6. Phase assemblages of

Table 1. Calculated composition and $\mathrm{pH}$ of the aqueous solution in equilibrium with selected solids in water, $25^{\circ} \mathrm{C}, 1 \mathrm{bar}$ pressure

\begin{tabular}{|c|c|c|c|c|c|c|}
\hline & $\mathrm{Ca}: \mathrm{mM} / \mathrm{kg}$ & $\mathrm{Al}: \mathrm{mM} / \mathrm{kg}$ & $\mathrm{Si}: \mathrm{mM} / \mathrm{kg}$ & $\mathrm{SO} 4: \mathrm{mM} / \mathrm{kg}$ & $\mathrm{CO} 3: \mathrm{mM} / \mathrm{Kg}$ & $\mathrm{pH}$ \\
\hline $\mathrm{C}-\mathrm{S}-\mathrm{H}(\mathrm{SI}) *$ & $1 \cdot 228$ & - & $1 \cdot 116$ & - & - & $11 \cdot 03$ \\
\hline $\mathrm{C}-\mathrm{S}-\mathrm{H}(\mathrm{SII})^{*}$ & $5 \cdot 212$ & - & $2 \cdot 895$ & - & - & $11 \cdot 69$ \\
\hline SH amorphous & - & - & $1 \cdot 413$ & - & - & $6 \cdot 37$ \\
\hline $\mathrm{C}_{2} \mathrm{ASH}_{8}$ & 0.769 & $0 \cdot 769$ & $0 \cdot 384$ & - & - & $10 \cdot 59$ \\
\hline Hydrogarnet Si low* & $2 \cdot 926$ & $1 \cdot 951$ & $0 \cdot 292$ & - & - & $11 \cdot 49$ \\
\hline Hydrogarnet Si high* & $2 \cdot 207$ & $1 \cdot 472$ & $0 \cdot 588$ & - & - & $11 \cdot 29$ \\
\hline $\mathrm{C}_{3} \mathrm{AH}_{6}$ & $3 \cdot 711$ & $2 \cdot 474$ & - & - & - & $11 \cdot 64$ \\
\hline $\mathrm{CH}$ & 21.95 & - & - & - & - & $12 \cdot 54$ \\
\hline $\mathrm{AH}_{3}$ (gibbsite) & - & $3 \cdot 25 \mathrm{E}-6$ & - & - & - & $6 \cdot 99$ \\
\hline Calcium monocarboaluminate $\dagger$ & $2 \cdot 561$ & $1 \cdot 280$ & - & - & 0.640 & $11 \cdot 37$ \\
\hline Calcium hemicarboaluminate $\dagger$ & $4 \cdot 110$ & $2 \cdot 055$ & - & - & $0 \cdot 514$ & $11 \cdot 65$ \\
\hline Calcite & $0 \cdot 146$ & - & - & - & $0 \cdot 146$ & $9 \cdot 96$ \\
\hline Ettringite & $1 \cdot 647$ & $0 \cdot 549$ & - & $0 \cdot 824$ & - & $11 \cdot 00$ \\
\hline Gypsum & $15 \cdot 26$ & - & - & $15 \cdot 26$ & - & $7 \cdot 07$ \\
\hline Thaumasite & 0.7144 & - & $0 \cdot 2382$ & $0 \cdot 2382$ & $0 \cdot 2382$ & $10 \cdot 50$ \\
\hline
\end{tabular}

* See text for definition of the formulae of these phases.

$\dagger$ Calcium hemicarboaluminate, $4 \mathrm{CaO} \cdot \mathrm{Al}_{2} \mathrm{O}_{3} \cdot 0 \cdot 5 \mathrm{CO}_{3} \cdot 12 \mathrm{H}_{2} \mathrm{O}$ and calcium monocarboaluminate, $4 \mathrm{CaO} \cdot \mathrm{Al}_{2} \mathrm{O}_{3} \cdot \mathrm{CO}_{3} \cdot 11 \mathrm{H}_{2} \mathrm{O}$. 
Table 4. Stable assemblages with three solids coexisting with an aqueous (aq) phase

PORTLAND $+\mathrm{C}_{3} \mathrm{AH}_{6}+$ SIHGlow + aq

PORTLAND $+\mathrm{C}_{3} \mathrm{AH}_{6}+$ ETTRINGITE + aq

PORTLAND $+\mathrm{C}_{3} \mathrm{AH}_{6}+$ HEMICARB + aq.

PORTLAND + SIHGlow + ETTRINGITE + aq

PORTLAND + SIHGlow + HEMICARB + aq.

PORTLAND + ETTRINGITE + HEMICARB + aq.

$\mathrm{C}_{3} \mathrm{AH}_{6}+$ SIHGlow + ETTRINGITE + aq.

$\mathrm{C}_{3} \mathrm{AH}_{6}+$ SIHGlow + HEMICARB + aq.

$\mathrm{C}_{3} \mathrm{AH}_{6}+$ ETTRINGITE + HEMICARB + aq.

SIHGlow + ETTRINGITE + HEMICARB + aq

$\mathrm{C}_{3} \mathrm{AH}_{6}+$ SIHGlow + MONOCARB + aq.

$\mathrm{C}_{3} \mathrm{AH}_{6}+$ ETTRINGITE + MONOCARB + aq.

$\mathrm{C}_{3} \mathrm{AH}_{6}+$ MONOCARB + HEMICARB + aq.

SIHGlow + ETTRINGITE + MONOCARB + aq.

SIHGlow + MONOCARB + HEMICARB + aq.

ETTRINGITE + MONOCARB + HEMICARB + aq.

PORTLAND + SIHGlow + CSH(SII) + aq.

PORTLAND + CSH(SII) + ETTRINGITE + aq.

PORTLAND + CSH(SII) + HEMICARB + aq.

SIHGlow + CSH(SII) + ETTRINGITE + aq.

SIHGlow + CSH(SII) + HEMICARB + aq.

CSH(SII) + ETTRINGITE + HEMICARB + aq.

SIHGlow + CSH(SII) + MONOCARB + aq

CSH(SII) + ETTRINGITE + MONOCARB + aq.

$\mathrm{CSH}(\mathrm{SII})+\mathrm{MONOCARB}+$ HEMICARB + aq.

SIHGlow + CSH(SI) + CSH(SII) + aq.

SIHGlow + CSH(SI) + ETTRINGITE + aq.

SIHGlow + CSH(SI) + MONOCARB + aq.

$\mathrm{CSH}(\mathrm{SI})+\mathrm{CSH}(\mathrm{SII})+$ ETTRINGITE + aq.

$\mathrm{CSH}(\mathrm{SI})+\mathrm{CSH}(\mathrm{SII})+\mathrm{MONOCARB}+\mathrm{aq}$

CSH(SI) + ETTRINGITE + MONOCARB + aq.

PORTLAND + CSH(SII) + MONOCARB + aq.

PORTLAND + ETTRINGITE + MONOCARB + aq.

PORTLAND + MONOCARB + HEMICARB + aq.

$\mathrm{C}_{3} \mathrm{AH}_{6}+$ SIHGlow + GIBBSITE + aq.

$\mathrm{C}_{3} \mathrm{AH}_{6}+$ GIBBSITE + ETTRINGITE + aq.

$\mathrm{C}_{3} \mathrm{AH}_{6}+$ GIBBSITE + MONOCARB + aq.

SIHGlow + GIBBSITE + ETTRINGITE + aq.

SIHGlow + GIBBSITE + MONOCARB + aq.

GIBBSITE + ETTRINGITE + MONOCARB + aq.

CALCITE + PORTLAND + CSH(SII) + aq.

CALCITE + PORTLAND + ETTRINGITE + aq.

CALCITE + PORTLAND + MONOCARB + aq.

CALCITE + CSH(SII) + ETTRINGITE + aq.

CALCITE + CSH(SII) + MONOCARB + aq.

CALCITE + ETTRINGITE + MONOCARB + aq.

CALCITE + CSH(SI) + CSH(SII $)+$ aq.

CALCITE + CSH(SI) + ETTRINGITE + aq.

CALCITE + CSH(SI) + MONOCARB + aq.

CALCITE + SIHGlow + CSH(SI) + aq.

CALCITE + SIHGlow + ETTRINGITE + aq.

CALCITE + SIHGlow + MONOCARB + aq.

CALCITE + SIHGlow + SIHGhigh + aq.

CALCITE + SIHGhigh + CSH(SI) + aq.

CALCITE + SIHGhigh + ETTRINGITE + aq

SIHGlow + SIHGhigh + CSH(SI) + aq.

SIHGlow + SIHGhigh + ETTRINGITE + aq.

SIHGhigh + CSH(SI) + ETTRINGITE + aq.

$\mathrm{C}_{2} \mathrm{ASH}_{8}+$ SIHGlow + GIBBSITE + aq

$\mathrm{C}_{2} \mathrm{ASH}_{8}+$ SIHGlow + ETTRINGITE + aq.

$\mathrm{C}_{2} \mathrm{ASH}_{8}+$ SIHGlow + MONOCARB + aq.
$\mathrm{C}_{2} \mathrm{ASH}_{8}+$ GIBBSITE + ETTRINGITE + aq.

$\mathrm{C}_{2} \mathrm{ASH}_{8}+$ GIBBSITE + MONOCARB + aq.

$\mathrm{C}_{2} \mathrm{ASH}_{8}+$ ETTRINGITE + MONOCARB + aq.

CALCITE $+\mathrm{C}_{2} \mathrm{ASH}_{8}+$ SIHGlow + aq.

CALCITE $+\mathrm{C}_{2} \mathrm{ASH}_{8}+$ ETTRINGITE + aq.

CALCITE $+\mathrm{C}_{2} \mathrm{ASH}_{8}+$ MONOCARB + aq.

CALCITE $+\mathrm{C}_{2} \mathrm{ASH}_{8}+$ SIHGhigh + aq.

$\mathrm{C}_{2} \mathrm{ASH}_{8}+$ SIHGlow + SIHGhigh + aq.

$\mathrm{C}_{2} \mathrm{ASH}_{8}+\mathrm{SIHGhigh}+$ ETTRINGITE + aq.

CALCITE $+\mathrm{C}_{2} \mathrm{ASH}_{8}+$ GIBBSITE + aq.

CALCITE + GIBBSITE + ETTRINGITE + aq.

CALCITE + GIBBSITE + MONOCARB + aq.

$\mathrm{CALCITE}+\mathrm{C}_{2} \mathrm{ASH}_{8}+\mathrm{CSH}(\mathrm{SI})+$ aq.

$\mathrm{C}_{2} \mathrm{ASH}_{8}+\mathrm{SIHGhigh}+\mathrm{CSH}(\mathrm{SI})+$ aq.

$\mathrm{C}_{2} \mathrm{ASH}_{8}+\mathrm{CSH}(\mathrm{SI})+$ ETTRINGITE + aq.

CALCITE + GIBBSITE + $\mathrm{SiO}_{2}$ gel + aq.

CALCITE + GIBBSITE + CSH(SI) + aq

CALCITE + GIBBSITE + THAUMASITE + aq.

$\mathrm{CALCITE}+\mathrm{SiO}_{2}$ gel $+\mathrm{CSH}(\mathrm{SI})+$ aq.

CALCITE $+\mathrm{SiO}_{2}$ gel + THAUMASITE + aq.

CALCITE + CSH(SI) + THAUMASITE + aq.

GIBBSITE $+\mathrm{SiO}_{2}$ gel $+\mathrm{CSH}(\mathrm{SI})+$ aq.

GIBBSITE $+\mathrm{SiO}_{2}$ gel + THAUMASITE + aq.

GIBBSITE + CSH(SI) + THAUMASITE + aq.

$\mathrm{SiO}_{2}$ gel $+\mathrm{CSH}(\mathrm{SI})+$ THAUMASITE + aq.

CALCITE + CSH(SII) + THAUMASITE + aq.

CALCITE + ETTRINGITE + THAUMASITE + aq.

$\mathrm{CSH}(\mathrm{SI})+\mathrm{CSH}(\mathrm{SII})+$ THAUMASITE + aq.

CSH(SI) + ETTRINGITE + THAUMASITE + aq.

CSH(SII) + ETTRINGITE + THAUMASITE + aq.

CALCITE $+\mathrm{C}_{2} \mathrm{ASH}_{8}+$ THAUMASITE + aq.

$\mathrm{C}_{2} \mathrm{ASH}_{8}+\mathrm{CSH}(\mathrm{SI})+$ THAUMASITE + aq.

$\mathrm{C}_{2} \mathrm{ASH}_{8}+$ ETTRINGITE + THAUMASITE + aq.

$\mathrm{C}_{2} \mathrm{ASH}_{8}+$ GIBBSITE $+\mathrm{CSH}(\mathrm{SI})+$ aq.

$\mathrm{C}_{2} \mathrm{ASH}_{8}+$ GIBBSITE + THAUMASITE + aq.

CALCITE + PORTLAND + THAUMASITE + aq.

PORTLAND + CSH(SII) + THAUMASITE + aq.

PORTLAND + ETTRINGITE + THAUMASITE + aq.

GIBBSITE + ETTRINGITE + THAUMASITE + aq.

GIBBSITE + CSH(SI) + ETTRINGITE + aq.

GYPSUM + PORTLAND + CSH(SII) + aq.

GYPSUM + PORTLAND + ETTRINGITE + aq.

GYPSUM + PORTLAND + THAUMASITE + aq.

GYPSUM + CSH(SII) + ETTRINGITE + aq.

GYPSUM + CSH(SII) + THAUMASITE + aq.

GYPSUM + ETTRINGITE + THAUMASITE + aq.

GYPSUM + CALCITE + PORTLAND + aq.

GYPSUM + CALCITE + ETTRINGITE + aq.

GYPSUM + CALCITE + THAUMASITE + aq.

GYPSUM + $\mathrm{CSH}(\mathrm{SI})+\mathrm{CSH}(\mathrm{SII})+$ aq.

GYPSUM + CSH(SI) + ETTRINGITE + aq.

GYPSUM + CSH(SI) + THAUMASITE + aq.

$\mathrm{GYPSUM}+\mathrm{GIBBSITE}+\mathrm{SiO}_{2} \mathrm{gel}+\mathrm{aq}$.

GYPSUM + GIBBSITE + CSH(SI) + aq.

GYPSUM + GIBBSITE + THAUMASITE + aq.

$\mathrm{GYPSUM}+\mathrm{SiO}_{2}$ gel $+\mathrm{CSH}(\mathrm{SI})+$ aq.

GYPSUM $+\mathrm{SiO}_{2}$ gel + THAUMASITE + aq.

GYPSUM + GIBBSITE + ETTRINGITE + aq.

GYPSUM + CALCITE + GIBBSITE + aq.

GYPSUM + CALCITE $+\mathrm{SiO}_{2}$ gel + aq.

See text and Tables 1 and 2 for definition of abbreviations. 
PORTLAND $+\mathrm{C}_{3} \mathrm{AH}_{6}+$ SIHGlow + ETTRINGITE + aq.

PORTLAND $+\mathrm{C}_{3} \mathrm{AH}_{6}+$ SIHGlow + HEMICARB + aq.

PORTLAND $+\mathrm{C}_{3} \mathrm{AH}_{6}+$ ETTRINGITE + HEMICARB + aq.

PORTLAND + SIHGlow + ETTRINGITE + HEMICARB + aq

$\mathrm{C}_{3} \mathrm{AH}_{6}+$ SIHGlow + ETTRINGITE + HEMICARB + aq.

$\mathrm{C}_{3} \mathrm{AH}_{6}+$ SIHGlow + ETTRINGITE + MONOCARB + aq.

$\mathrm{C}_{3} \mathrm{AH}_{6}+$ SIHGlow + MONOCARB + HEMICARB + aq.

$\mathrm{C}_{3} \mathrm{AH}_{6}+$ ETTRINGITE + MONOCARB + HEMICARB + aq.

SIHGlow + ETTRINGITE + MONOCARB + HEMICARB + aq. PORTLAND + SIHGlow + CSH(SII) + ETTRINGITE + aq. PORTLAND + SIHGlow + CSH(SII) + HEMICARB + aq. PORTLAND + CSH(SII) + ETTRINGITE + HEMICARB + aq. SIHGlow + CSH(SII) + ETTRINGITE + HEMICARB + aq. SIHGlow + CSH(SII) + ETTRINGITE + MONOCARB + aq SIHGlow + CSH(SII) + MONOCARB + HEMICARB + aq. CSH(SII) + ETTRINGITE + MONOCARB + HEMICARB + aq. SIHGlow + CSH(SI) + CSH(SII) + ETTRINGITE + aq SIHGlow + CSH(SI) + CSH(SII) + MONOCARB + aq. SIHGlow + CSH(SI) + ETTRINGITE + MONOCARB + aq. $\mathrm{CSH}(\mathrm{SI})+\mathrm{CSH}(\mathrm{SII})+$ ETTRINGITE + MONOCARB + aq. PORTLAND + CSH(SII) + ETTRINGITE + MONOCARB + aq. PORTLAND + CSH(SII) + MONOCARB + HEMICARB + aq. PORTLAND + ETTRINGITE + MONOCARB + HEMICARB + aq. $\mathrm{C}_{3} \mathrm{AH}_{6}+$ SIHGlow + GIBBSITE + ETTRINGITE + aq. $\mathrm{C}_{3} \mathrm{AH}_{6}+$ SIHGlow + GIBBSITE + MONOCARB + aq. $\mathrm{C}_{3} \mathrm{AH}_{6}+$ GIBBSITE + ETTRINGITE + MONOCARB + aq. SIHGlow + GIBBSITE + ETTRINGITE + MONOCARB + aq. CALCITE + PORTLAND + CSH(SII) + ETTRINGITE + aq. CALCITE + PORTLAND + CSH(SII) + MONOCARB + aq. CALCITE + PORTLAND + ETTRINGITE + MONOCARB + aq CALCITE + CSH(SII) + ETTRINGITE + MONOCARB + aq. CALCITE + CSH(SI) + CSH(SII) + ETTRINGITE + aq. $\mathrm{CALCITE}+\mathrm{CSH}(\mathrm{SI})+\mathrm{CSH}(\mathrm{SII})+\mathrm{MONOCARB}+\mathrm{aq}$. CALCITE + CSH(SI) + ETTRINGITE + MONOCARB + aq. CALCITE + SIHGlow + CSH(SI) + ETTRINGITE + aq. CALCITE + SIHGlow + CSH(SI) + MONOCARB + aq. CALCITE + SIHGlow + ETTRINGITE + MONOCARB + aq. CALCITE + SIHGlow + SIHGhigh + CSH(SI) + aq. CALCITE + SIHGlow + SIHGhigh + ETTRINGITE + aq. CALCITE + SIHGhigh + CSH(SI) + ETTRINGITE + aq. SIHGlow + SIHGhigh + CSH(SI) + ETTRINGITE + aq. $\mathrm{C}_{2} \mathrm{ASH}_{8}+$ SIHGlow + GIBBSITE + ETTRINGITE + aq. $\mathrm{C}_{2} \mathrm{ASH}_{8}+$ SIHGlow + GIBBSITE + MONOCARB + aq. $\mathrm{C}_{2} \mathrm{ASH}_{8}+$ SIHGlow + ETTRINGITE + MONOCARB + aq. $\mathrm{C}_{2} \mathrm{ASH}_{8}+$ GIBBSITE + ETTRINGITE + MONOCARB + aq. CALCITE $+\mathrm{C}_{2} \mathrm{ASH}_{8}+$ SIHGlow + ETTRINGITE + aq. CALCITE $+\mathrm{C}_{2} \mathrm{ASH}_{8}+$ SIHGlow + MONOCARB + aq. CALCITE $+\mathrm{C}_{2} \mathrm{ASH}_{8}+$ ETTRINGITE + MONOCARB + aq. CALCITE $+\mathrm{C}_{2} \mathrm{ASH}_{8}+$ SIHGlow + SIHGhigh + aq. CALCITE $+\mathrm{C}_{2} \mathrm{ASH}_{8}+$ SIHGhigh + ETTRINGITE + aq.
$\mathrm{C}_{2} \mathrm{ASH}_{8}+$ SIHGlow + SIHGhigh + ETTRINGITE + aq. CALCITE $+\mathrm{C}_{2} \mathrm{ASH}_{8}+$ GIBBSITE + ETTRINGITE + aq. CALCITE $+\mathrm{C}_{2} \mathrm{ASH}_{8}+$ GIBBSITE + MONOCARB + aq. CALCITE + GIBBSITE + ETTRINGITE + MONOCARB + aq. CALCITE $+\mathrm{C}_{2} \mathrm{ASH}_{8}+$ SIHGhigh $+\mathrm{CSH}(\mathrm{SI})+$ aq. CALCITE $+\mathrm{C}_{2} \mathrm{ASH}_{8}+\mathrm{CSH}(\mathrm{SI})+$ ETTRINGITE + aq. $\mathrm{C}_{2} \mathrm{ASH}_{8}+$ SIHGhigh + CSH(SI) + ETTRINGITE + aq. $\mathrm{CALCITE}+\mathrm{GIBBSITE}+\mathrm{SiO}_{2}$ gel $+\mathrm{CSH}(\mathrm{SI})+$ aq CALCITE + GIBBSITE $+\mathrm{SiO}_{2}$ gel + THAUMASITE + aq. CALCITE + GIBBSITE + CSH(SI) + THAUMASITE + aq. $\mathrm{CALCITE}+\mathrm{SiO}_{2}$ gel $+\mathrm{CSH}(\mathrm{SI})+$ THAUMASITE + aq. GIBBSITE $+\mathrm{SiO}_{2}$ gel $+\mathrm{CSH}(\mathrm{SI})+$ THAUMASITE + aq. CALCITE + CSH(SI $)+$ CSH(SII) + THAUMASITE + aq. CALCITE + CSH(SI) + ETTRINGITE + THAUMASITE + aq. CALCITE + CSH(SII) + ETTRINGITE + THAUMASITE + aq. $\mathrm{CSH}(\mathrm{SI})+\mathrm{CSH}(\mathrm{SII})+$ ETTRINGITE + THAUMASITE + aq. CALCITE $+\mathrm{C}_{2} \mathrm{ASH}_{8}+\mathrm{CSH}(\mathrm{SI})+$ THAUMASITE + aq. CALCITE $+\mathrm{C}_{2} \mathrm{ASH}_{8}+$ ETTRINGITE + THAUMASITE + aq. $\mathrm{C}_{2} \mathrm{ASH}_{8}+\mathrm{CSH}(\mathrm{SI})+$ ETTRINGITE + THAUMASITE + aq. $\mathrm{CALCITE}+\mathrm{C}_{2} \mathrm{ASH}_{8}+$ GIBBSITE $+\mathrm{CSH}(\mathrm{SI})+$ aq CALCITE $+\mathrm{C}_{2} \mathrm{ASH}_{8}+$ GIBBSITE + THAUMASITE + aq. $\mathrm{C}_{2} \mathrm{ASH}_{8}+$ GIBBSITE $+\mathrm{CSH}(\mathrm{SI})+$ THAUMASITE + aq. CALCITE + PORTLAND + CSH(SII) + THAUMASITE + aq. CALCITE + PORTLAND + ETTRINGITE + THAUMASITE + aq. PORTLAND + CSH(SII) + ETTRINGITE + THAUMASITE + aq. CALCITE + GIBBSITE + ETTRINGITE + THAUMASITE + aq. $\mathrm{C}_{2} \mathrm{ASH}_{8}+$ GIBBSITE + ETTRINGITE + THAUMASITE + aq. $\mathrm{C}_{2} \mathrm{ASH}_{8}+$ GIBBSITE $+\mathrm{CSH}(\mathrm{SI})+$ ETTRINGITE + aq. GIBBSITE + CSH(SI) + ETTRINGITE + THAUMASITE + aq. GYPSUM + PORTLAND + CSH(SII) + ETTRINGITE + aq. GYPSUM + PORTLAND + CSH(SII) + THAUMASITE + aq. GYPSUM + PORTLAND + ETTRINGITE + THAUMASITE + aq. GYPSUM + CSH(SII) + ETTRINGITE + THAUMASITE + aq. GYPSUM + CALCITE + PORTLAND + ETTRINGITE + aq. GYPSUM + CALCITE + PORTLAND + THAUMASITE + aq. GYPSUM + CALCITE + ETTRINGITE + THAUMASITE + aq GYPSUM + CSH(SI) + CSH(SII) + ETTRINGITE + aq. GYPSUM + CSH(SI) + CSH(SII) + THAUMASITE + aq. GYPSUM + CSH(SI) + ETTRINGITE + THAUMASITE + aq. $\mathrm{GYPSUM}+\mathrm{GIBBSITE}+\mathrm{SiO}_{2} \mathrm{gel}+\mathrm{CSH}(\mathrm{SI})+$ aq. GYPSUM + GIBBSITE + $\mathrm{SiO}_{2}$ gel + THAUMASITE + aq. GYPSUM + GIBBSITE + CSH(SI) + THAUMASITE + aq. GYPSUM $+\mathrm{SiO}_{2}$ gel + CSH(SI) + THAUMASITE + aq. GYPSUM + GIBBSITE + CSH(SI) + ETTRINGITE + aq. GYPSUM + GIBBSITE + ETTRINGITE + THAUMASITE + aq. GYPSUM + CALCITE + GIBBSITE + $\mathrm{SiO}_{2}$ gel + aq. GYPSUM + CALCITE + GIBBSITE + THAUMASITE + aq. GYPSUM + CALCITE $+\mathrm{SiO}_{2}$ gel + THAUMASITE + aq. GYPSUM + CALCITE + GIBBSITE + ETTRINGITE + aq.

See text and Tables 1 and 2 for definition of abbreviations.

more than two solids, but which include only two (or more) of the incompatible solids shown in Table 6 will also not be stable.

The calculations reveal many more phase assemblages than have been found experimentally or are recorded from structures undergoing TSA. However, we can immediately compare results with the limited data recorded in the literature. For example, and contrary to the results of Juel et al., the phase assemblage thaumasite + calcite + gypsum + ettringite is stable; indeed, this phase assemblage is often noted to occur in cement and concrete undergoing TSA. ${ }^{1}$

\section{Discussion}

In calculating equilibria in cement systems, certain phases known to be thermodynamically unstable are nevertheless very persistent. For example, C-S-H, the principal-binding phase of cements, is undoubtedly thermodynamically unstable with respect to crystalline $\mathrm{CaO}-\mathrm{SiO}_{2}-\mathrm{H}_{2} \mathrm{O}$ phases. However, we know that the $\mathrm{C}-\mathrm{S}-\mathrm{H}$ gel is important to the constitution of real cement and concretes. Unaltered historic Portland cements still contain $\mathrm{C}-\mathrm{S}-\mathrm{H}$ and it has been reported to occur in Roman concretes as well as in nature, in 
Table 6. Solid phase pairs in the $\mathrm{CaO}-\mathrm{Al}_{2} \mathrm{O}_{3}-\mathrm{SiO}_{2}-\mathrm{CaSO}_{4}-\mathrm{CaCO}_{3}-\mathrm{H}_{2} \mathrm{O}$ system at $25^{\circ} \mathrm{C}$ which, from calculation, are not compatible

\begin{tabular}{|c|c|c|}
\hline 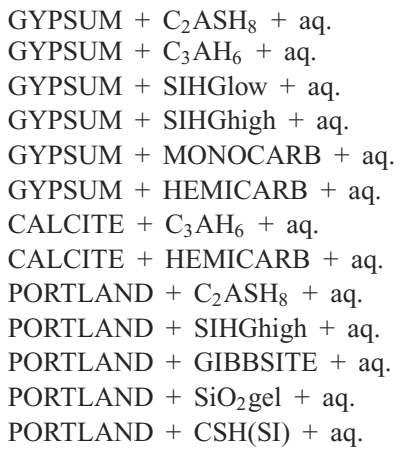 & 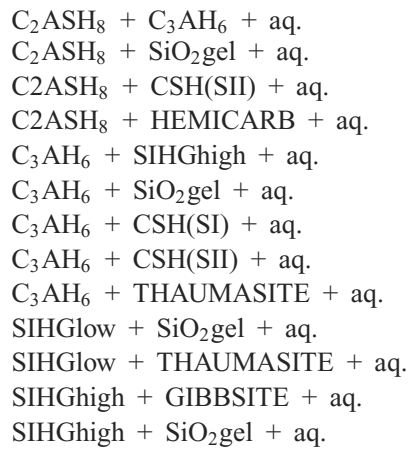 & $\begin{array}{l}\text { SIHGhigh + CSH(SII) + aq. } \\
\text { SIHGhigh + MONOCARB + aq. } \\
\text { SIHGhigh + HEMICARB + aq. } \\
\text { SIHGhigh + THAUMASITE + aq. } \\
\text { GIBBSITE + CSH(SII) + aq. } \\
\text { GIBBSITE + HEMICARB + aq. } \\
\mathrm{SiO}_{2} \text { gel + CSH(SII) + aq. } \\
\mathrm{SiO}_{2} \text { gel + ETTRINGITE + aq. } \\
\mathrm{SiO}_{2} \text { gel + MONOCARB + aq. } \\
\mathrm{SiO}_{2} \text { gel + HEMICARB + aq. } \\
\mathrm{CSH} \text { SI }+ \text { HEMICARB + aq. } \\
\mathrm{MONOCARB} \mathrm{+} \mathrm{THAUMASITE} \mathrm{+} \mathrm{aq.}_{\text {HEMICARB + THAUMASITE + aq. }} \text {. }\end{array}$ \\
\hline
\end{tabular}

hydrothermally-altered impure limestones. If calculations are to be relevant to real cements, it is essential to include these phases. Thus with the conspicuous exception of $\mathrm{C}-\mathrm{S}-\mathrm{H}$, which is allowed to replace the stable crystalline $\mathrm{C}-\mathrm{S}-\mathrm{H}$ minerals, and silica gel, allowed to replace quartz, the calculations are otherwise allowed to proceed on the basis of true thermodynamic equilibrium. Since thaumasite mainly appears in altered concretes, and since the driving force for chemical and mineralogical reconstitution of cement paste mineralogy is the approach to thermodynamic equilibrium, the authors see no inconsistency in the approach taken, namely: of using equilibrium but admitting selected metastable but persistent phases into the scheme of calculation.

At the temperature used for the calculation $\left(25^{\circ} \mathrm{C}\right)$, several AFm minerals observed in cement are not thermodynamically stable. Examples include hydroxyand sulphate-containing AFm phases. For this reason, they are not included in the calculations whereas other stable AFm phases are included, for example $\mathrm{C}_{2} \mathrm{ASH}_{8}$, mono- and hemicarboaluminate. The mineralogy of the system is very sensitive to temperature. At some temperature, probably not much exceeding $25^{\circ} \mathrm{C}$, thaumasite will no longer be stable. On the other hand, at temperatures exceeding $\sim 40^{\circ} \mathrm{C}$, sulphate-AFm becomes thermodynamically stable and thus the range of stable AFm chemistries will broaden. The authors do not at present have the data necessary to repeat the calculations undertaken here at other temperatures, especially at $>25^{\circ} \mathrm{C}$, but as part of a wider study of the relationships between cement paste mineralogy, temperature, environmentally-conditioned reactions and durability of the resulting concrete, the quickest way to establish quantitative links with paste mineralogy may be to identify and subsequently obtain and apply the missing data.

Some ranges of solid solution, for example between ettringite and thaumasite, have also not been included in the calculation. The impact of solid solution will be, in general, to enhance the importance of phase assem- blages containing fewer than the maximum permitted number of phases. Thus sulphate, carbonate and silicate concentrations, which are predicted in the model to form assemblages containing both ettringite and thaumasite may, in practice, give only one phase, typically an ettringite solid solution. These data deficiencies do not invalidate the general conclusions reached here but simply shift the boundaries between phase regions mainly by extending the stability domain of ettringite.

Results of the calculation disclose that thaumasite is stable at $25^{\circ} \mathrm{C}$. The question of the kinetics of its formation is not handled by thermodynamics, but once formed, typically but not necessarily exclusively at $<25^{\circ} \mathrm{C}$, it can persist stably over a broad temperature. This is supported by field experiments ${ }^{2}$ and by natural occurrences of thaumasite. The thaumasite stability domain is wide and, as a consequence, thaumasite coexists stably with all the phases of the $\mathrm{CaO}-\mathrm{Al}_{2} \mathrm{O}_{3}-$ $\mathrm{SiO}_{2}-\mathrm{CaSO}_{4}-\mathrm{CaCO}_{3}-\mathrm{H}_{2} \mathrm{O}$ system with the exception of hydrogarnets $\left(\mathrm{C}_{3} \mathrm{AH}_{6}, \mathrm{HG} \mathrm{Si}\right.$ low and $\mathrm{HG} \mathrm{Si}$ high $)$ and with calcium hemicarboaluminate and monocarboaluminate. The incompatibility of assemblages containing carboaluminates is in agreement with the calculation of Juel et $\mathrm{al}^{7}$ Hydrogarnet and Si-substituted hydrogarnet are not stable with thaumasite; they react, with transformation to ettringite, while the sulphate concentration in the aqueous phase is still insufficient to stabilise thaumasite. Indeed, if the invariant points are sorted in order of increasing sulphate concentration of the aqueous phase necessary to stabilise the various solid phase assemblages, as has been done in Table 2, the first 16 invariant points do not contain thaumasite but instead contain ettringite. This arises because thaumasite characteristically requires higher sulfate concentrations than ettringite to form. Ettringite is however frequently found to occur with thaumasite. The four invariant points, numbered 17, 20, 27 and 29 in Table 2, are exceptions: gibbsite is always present and the $\mathrm{pH}$ is below 12. These four invariant points represent two different chemical environments: invariant points 17 and 20 correspond to low 
sulphate concentrations whereas 27 and 29 correspond to characteristically high sulphate concentrations conditioned by the solubility of gypsum. In these conditions thaumasite can form directly without the need for concomitant or anterior formation of ettringite. This is considered to resolve the controversy concerning the need to have ettringite as a precursor for thaumasite formation; while this may occur under some conditions it is not a prerequisite in all ranges of composition. The invariant point 29 , of gypsum + calcite + gibbsite + $\mathrm{SiO}_{2}$ gel + thaumasite, is also very interesting: first because it is supposed to be the final stage of TSA, and second, because it corresponds to a quite low $\mathrm{pH}$ aqueous (8.51). Thus while ettringite cannot be in equilibrium with $\mathrm{pH}$ lower than about 9.5 (point 30 ), thaumasite can exist stably to lower $\mathrm{pH}$. This is in disagreement with Gaze and Crammond ${ }^{1}$ who report that thaumasite does not form at $\mathrm{pH}$ less than 10.5: the current authors fix the limit as approximately one $\mathrm{pH}$ unit lower. Also, the conclusions reported by Jallad et $a l .{ }^{15}$ seem to be more related to the addition of other ions used to control the $\mathrm{pH}$ rather than to the stability of thaumasite itself.

It should also be noted that high aqueous carbonate contents are not a necessary prerequisite for formation of thaumasite. For example, at invariant point 28 , Table 2 , the composition of the aqueous solution is calculated to contain $2.53 \times 10^{-8} \mathrm{~mol} / \mathrm{kg}$ of carbonate. Only when calcite occurs together with thaumasite, as at invariant points 17, 18, 19, 20, 21, 22, 25, 29 and 30, do aqueous carbonate contents increase to $\sim 10^{-5} \mathrm{~mol} / \mathrm{kg}$, sustained by the relatively high solubility of calcite. While some of the assemblages listed above are only likely to occur in highly altered cements, thaumasite can coexist with $\mathrm{C}-\mathrm{S}-\mathrm{H}$ as for example in assemblages 18, 19, 21 and 24, Table 2. These assemblages might appear in plain or blended cements relatively early in their history of alteration.

Broad consideration of the results shown in the tables, coupled with experience of cements and concrete undergoing chemical alteration, either as a result of internal reaction or reaction with their service environment, suggests that with few exceptions the driving force for chemical-mineralogical alteration results from an approach to a local thermodynamic equilibrium. The exceptions include the persistence of a few well-defined but metastable phases such as C-S$\mathrm{H}$. While the data given here define all energetically feasible assemblages at $25^{\circ} \mathrm{C}$, the reaction pathways of cement undergoing reaction with its service environment are not intuitively obvious. Reaction pathways are determined in practice by a combination of dissolution and reaction: in general, the dissolution component remains unknown in field studies. We need patiently to relate microscopic and diffusion studies to the phase equilibria in order to determine the response of paste mineralogy to diffusional composition gradients in order to infer to what extent dissolution has occurred.
We need additionally to improve the accuracy of thermodynamic predictions, as for example by determining more precisely the extent of solid solutions and of element fractionation between coexisting phases. We also need increasingly to apply thermodynamics, coupled with geochemical limitations on, for example, natural ground water compositions, to devise standard test methods which more accurately reflect the performance of cement concretes in adverse environments Above all, we need to quantify deteriorations, so as to be able to produce tailored cement and concrete formulations which will give enhanced performance in adverse service environments. Decades of largely empirical research have produced considerable enhancement in concrete durability but the authors submit that a coupled chemical thermodynamic-property model represents the way forward.

\section{Conclusion}

The phase diagram of the $\mathrm{CaO}-\mathrm{Al}_{2} \mathrm{O}_{3}-\mathrm{SiO}_{2}-$ $\mathrm{CaSO}_{4}-\mathrm{CaCO}_{3}-\mathrm{H}_{2} \mathrm{O}$ system has been resolved by calculation of the composition of the stable invariant points. The results demonstrate the usefulness of this approach for the investigation complex of multicomponent systems.

Thaumasite is calculated to be stable over a wide range of aqueous phase compositions and is one of the major phases of the $\mathrm{CaO}-\mathrm{Al}_{2} \mathrm{O}_{3}-\mathrm{SiO}_{2}-\mathrm{CaSO}_{4}-$ $\mathrm{CaCO}_{3}-\mathrm{H}_{2} \mathrm{O}$ system at $25^{\circ} \mathrm{C}$. With respect to ettringite, thaumasite formation generally demands more sulphate ions in the aqueous phase but thaumasite is also stable at $\mathrm{pH}$ as low as 8.5 compared to 9.5 for ettringite. In these low $\mathrm{pH}$ conditions, thaumasite is expected to form directly without need for the formation of precursor ettringite. These conditions are believed to be responsible for much of the damage occurring during TSA. The phase development model presented here provides a flexible tool with which to predict chemical fluxes in cements undergoing alteration and relate chemical-mineralogical change to engineering properties.

\section{Acknowledgements}

The EPSRC (Engineering and Physical Sciences Research Council) is acknowledged for its support of D. E. MacPhee and S. J. Barnett.

\section{References}

1. Gaze M. and Crammond N. J. The formation of thaumasite in a cement:lime:sand mortar exposed to cold magnesium and potassium sulfate solutions. Cement and Concrete Research, 2000, 30, 209-222. 
2. SAhu S., Badger S. and Thaulow N. Evidence of thaumasite formation in southern California concrete. Cement and Concrete Composites, 2002, 24, 379-384.

3. Damidot D. and Glasser F. P. Thermodynamic investigation of the $\mathrm{CaO}-\mathrm{Al}_{2} \mathrm{O}_{3}-\mathrm{CaSO}_{4}-\mathrm{H}_{2} \mathrm{O}$ system at $25^{\circ} \mathrm{C}$ and influence of $\mathrm{Na}_{2} \mathrm{O}$. Cement and Concrete Research, 1993, 23, 221-238.

4. Damidot D., Stronach S., Kindness A., Atkins M. and Glasser F. P. Thermodynamic investigation of the $\mathrm{CaO}-$ $\mathrm{Al}_{2} \mathrm{O}_{3}-\mathrm{CaCO}_{3}-\mathrm{H}_{2} \mathrm{O}$ system at $25^{\circ} \mathrm{C}$ and the influence of $\mathrm{Na}_{2} \mathrm{O}$. Cement and Concrete Research, 1994, 24, 563-572.

5. Stronach S. and Glasser F. P. Modelling the impact of abundant geochemical components on phase stability and solubility of the $\mathrm{CaO}-\mathrm{SiO}_{2}-\mathrm{H}_{2} \mathrm{O}$ system at $25^{\circ} \mathrm{C}: \mathrm{Na}^{+}, \mathrm{K}^{+}$, $\mathrm{SO}_{4}{ }^{2-}, \mathrm{Cl}^{-}$and $\mathrm{CO}_{3}{ }^{2-}$. Advances in Cement Research, 1997, 9, No. 36, 167-182.

6. Damidot D. and Glasser F. P. Investigation of the $\mathrm{CaO}-$ $\mathrm{Al}_{2} \mathrm{O}_{3}-\mathrm{SiO}_{2}-\mathrm{H}_{2} \mathrm{O}$ system at $25^{\circ} \mathrm{C}$ by thermodynamic calculations. Cement and Concrete Research, 1995, 25, 22-28.

7. Juel I., Herfort D., Gollop R., Konnerup-Madsen J., JAKOBSEN H. J. and SkiBSted J. A thermodynamic model for predicting the stability of thaumasite. Proceedings of the Ist International Conference on Thaumasite in Cementitious Materials, Watford, UK, 19-21 June 2002.

8. Edge R. A. and TAYLOR H. F. W. Crystal structure of thaumasite $\left[\mathrm{Ca}_{3} \mathrm{Si}(\mathrm{OH})_{6} \cdot 12 \mathrm{H}_{2} \mathrm{O}\right] \mathrm{SO}_{4} \cdot \mathrm{CO}_{3}$. Acta Crystallographa, 1971, B27, 594-601.

9. Barnett S. J., Macphee D. E., Lachowski E. E. and Crammond N. J. XRD, EDX and IR analysis of solid solutions between thaumasite and ettringite. Cement and Concrete Research, 2002, 32, 719-730.

10. Plummer L. N., Parkhurst D. L., Fleming G. W. and Dunkle S. A. A Computer Program Incorporating Pitzer's Equations for Calculation of Geochemical Reactions in Brines. US Geological, Survey Water-Resources Investigations Report 88-4153, 1988.

11. Pitzer K. S. Thermodynamics of electrolytes I: theoretical basis and general equations. Journal of Physical Chemistry, 1973, 77, 268-277.

12. Nonat A., Courault A.-C. and Damidot D. A surface reaction model describing the structure and stoichiometry variations of calcium silicate hydrate $(\mathrm{C}-\mathrm{S}-\mathrm{H})$ with calcium hydroxide concentration in solution. Langmuir, forthcoming.

13. DAmidot D. and NonAt A. $\mathrm{C}_{3} \mathrm{~S}$ hydration in diluted and stirred suspension: (II) Properties of C-S-H precipitated during the two kinetic steps. Advances in Cement Research, 1994, 22, 83-91.

14. JAPPY T. G. and Glasser F. P. Synthesis and stability of silica substituted hydrogarnet, $\mathrm{Ca}_{3} \mathrm{Al}_{2} \mathrm{Si}_{3-\mathrm{x}} \mathrm{O}_{12-4} \mathrm{x}(\mathrm{OH})_{4 \mathrm{x}}$. Advances in Cement Research, 1991, 4, No. 1, 1-8.

15. Jallad K. N., Santhanam M. and Cohen M. D. Stability and reactivity of thaumasite at different $\mathrm{pH}$ levels. Cement and Concrete Research, 2000, 33, 433-437. 